Ann. Biol. anim. Bioch. Biophys., 1977, 17 (6), 1043-1046.

\title{
Construction de minivannes programmables
}

par Y. BONNET

Laboratoire d'Efude du Méfabolisme Azofé, I. N. R. A., Theix, Saint Genès Champanelle 63110 Beaumont.

Summary. Construction of programmable microvalves.

Cam-activated microswitches were modified to open or close plastic or rubber tubes. They may be used as corrosion-proof microvalves on chromatographic or other devices. The same programmers may be used to activate either microswitches or microvalves or combinations of both.

\section{Principe.}

Les différentes manipulations pratiquées tant au laboratoire (chromatographie, analyses diverses utilisant un ou plusieurs réactifs) que sur animaux (infusions, prélèvements) nécessitent souvent l'emploi d'un dispositif permettant de sélectionner successivement différents fluides et d'en arrêter l'écoulement à la fin de l'analyse ou de l'expérience. Nous avons réalisé un dispositif simple et robuste, basé sur une modification des minirupteurs « Crouzet * » (type 83111-5) consistant à transformer ceuxci en vannes 2 voies programmables. Ces vannes utilisables dans le cas de débits et de pressions relativement faibles fonctionnent par étranglement de tuyau plastique souple (ce matériel ne peut donc pas être utilisé pour de la commande pneumatique et $n$ 'est donc pas comparable aux minivannes actuellement commercialisées et actionnées de la même façon).

Elles s'apparentent, en ce qui concerne leur utilisation, aux électrovannes, en présentant les avantages suivants :

- conservation de l'état « ouvert» ou « fermé », en cas de coupure de courant ;

- absence de contact du fluide avec les parties mobiles du système (un choix judicieux de la nature du tuyau en fonction des fluides utilisés permettant généralement d'éliminer les problèmes de corrosion) ;

- continuité géométrique du circuit ;

* Crouzet S. A. - 128, avenue de la République, 75011 Paris.

Catalogue général division composants d'automatisme : fascicule 30-1, interrupteurs sensibles, fascicule 11-programmateurs à cames. 
- simplification des montages et économie appréciable (moins de composants, bas prix de revient de la vanne).

Ces vannes peuvent être actionnées manuellement ou par des cames.

Dans le cas du montage représenté figure 1, l'ouverture et la fermeture des vannes est assurée par un programmateur Crouzet type 221-1 entraîné par un motoréducteur.

Des minirupteurs affectés au circuit d'alimentation du motoréducteur arrêtent la rotation du programmateur lorsque les cames qui les actionnent provoquent leur inversion. Ceci permet à chaque alimentation du motoréducteur, provoquée par. un dispositif extérieur, de faire tourner le programmateur d'une fraction de tour au cours de laquelle des vannes peuvent être ouvertes ou fermées.

\section{Choix du motoréducteur.}

Dans le cas où l'ouverture et la fermeture des vannes sont assurées par un programmateur Crouzet type 221-1 (fig. 1), le couple nécessaire à la fermeture d'une vanne équipée d'un tuyau en caoutchouc siliconné est de 5 à $6 \mathrm{~cm}$ newton. Les programmateurs, actionnés par des motoréducteurs synchrones, sont équipés de cames réglables et de cames dont la forme peut être adaptée aux besoins par découpe de leur périphérie (de 5 voies maximum à 18 voies maximum selon le modèle). Les rampes de cames ouvrent ou ferment les vannes sur un parcours angulaire de l'ordre de

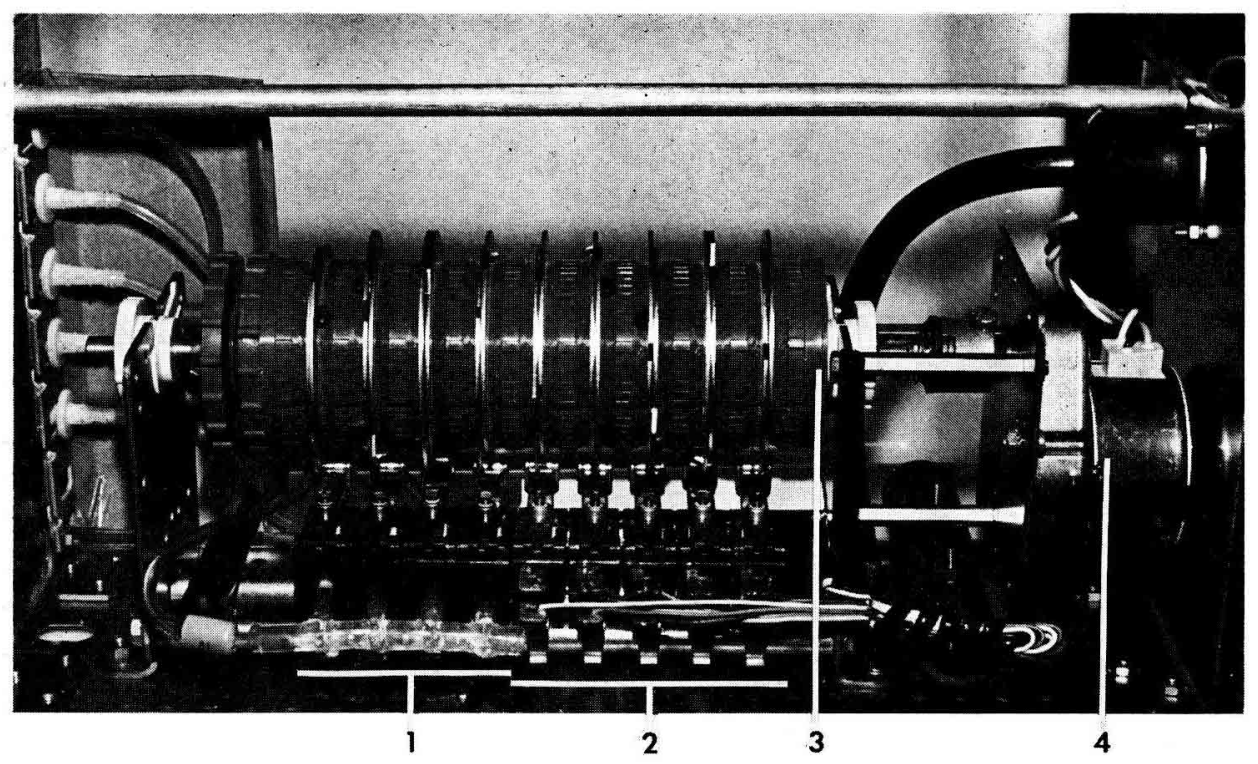

FIG. 1. - Programmateur équipé de minivannes el minirupteurs.

1. - 4 minivannes

2. -5 minirupteurs

3. - Tambour supportant 9 cames

4. - Motoréducteur 
10 degrés correspondant à $1 / 36$ e du temps de rotation du motoréducteur. II faut donc utiliser des vitesses de rotation relativement élevées, de l'ordre de 1 à 4 tours par minute, pour réduire les durées des états transitoires à des valeurs comprises entre 1,7 ef 0,4 secondes.

Le motoréducteur utilisé doit avoir, à cette vitesse, un couple suffisant pour actionner l'ensemble des vannes à fermer et des contacts électriques à inverser simultanément.

\section{Caractéristiques d'une vanne.}

Des mesures effectuées sur une vanne équipée de tuyau siliconé indiquent les ordres de grandeur suivants pour les débits :

$\begin{array}{lllll}\text { Pressions (bars) } \ldots \ldots \ldots \ldots & 0,25 & 0,5 & 0,75 \\ \text { Débit d'air }\left(\mathrm{m}^{3} / \mathrm{h}\right) \ldots \ldots \ldots \ldots & 0,8 & 1,2 & 1,5 \\ \text { Débit d'eau }(\mathrm{l} / \mathrm{h}) & \ldots \ldots \ldots \ldots & 30 & 45 & 55\end{array}$

La pression de 0,75 bar correspond à l'effort maximum admissible sur les axes du levier de manœuvre, et par conséquent aux débits maximum.

\section{Réalisation.}

Nous utilisons un minirupleur type $83111-5$ compatible avec le programmateur type 221-1. Le minirupteur est constitué d'un boîtier, un couvercle, un élément de commande (poussoir plastique mobilisant un équipage d'inversion de contacts, quatre pièces métalliques de contact et raccordement électrique), un levier de manœuvre muni d'une vis de réglage de course du poussoir, deux clips pour bridage sur programmateur. Deux rivets en aluminium maintiennent le couvercle solidaire du corps et le positionnement des autres constituants. Une lame de couteau insérée entre le boîtier et le couvercle puis manœuvrée comme un levier suffit pour désolidariser l'ensemble. Les 4 pièces de contact et l'équipage d'inversion sont supprimés. Tous les autres éléments sont utilisés.

Le boîtier est alésé au diamètre de $4 \mathrm{~mm}$ à ses deux extrémités selon son grand axe pour ménager un passage au tuyau souple qui le traverse complètement. La saillie interne du couvercle est rainurée pour le guidage du presseur de tuyau. Le presseur et une plaquette d'appui du tuyau sont réalisés dans du feuillard d'acier (inox de préférence) (fig. 2). La nature du tuyau (diamètre externe $4 \mathrm{~mm}$ ) est choisie en fonction de sa résistance chimique au fluide utilisé.

La plaquette d'appui est insérée entre le plancher du boîtier et le tuyau. L'ensemble poussoir et presseur remplaçant l'équipage d'inversion, est placé dans le boîtier. Ces opérations sont facilitées en tendant le fuyau et en lui imprimant un mouvement de rotation. Le levier de manœuvre ef les clips destinés au bridage sur le programmateur sont présentés dans leurs logements. Il est nécessaire de présenter le guide de l'élément presseur en face de la rainure de guidage avant de fermer le boîtier au moyen du couvercle qui est maintenu grâce à la mise en place des deux rivets en aluminium. 


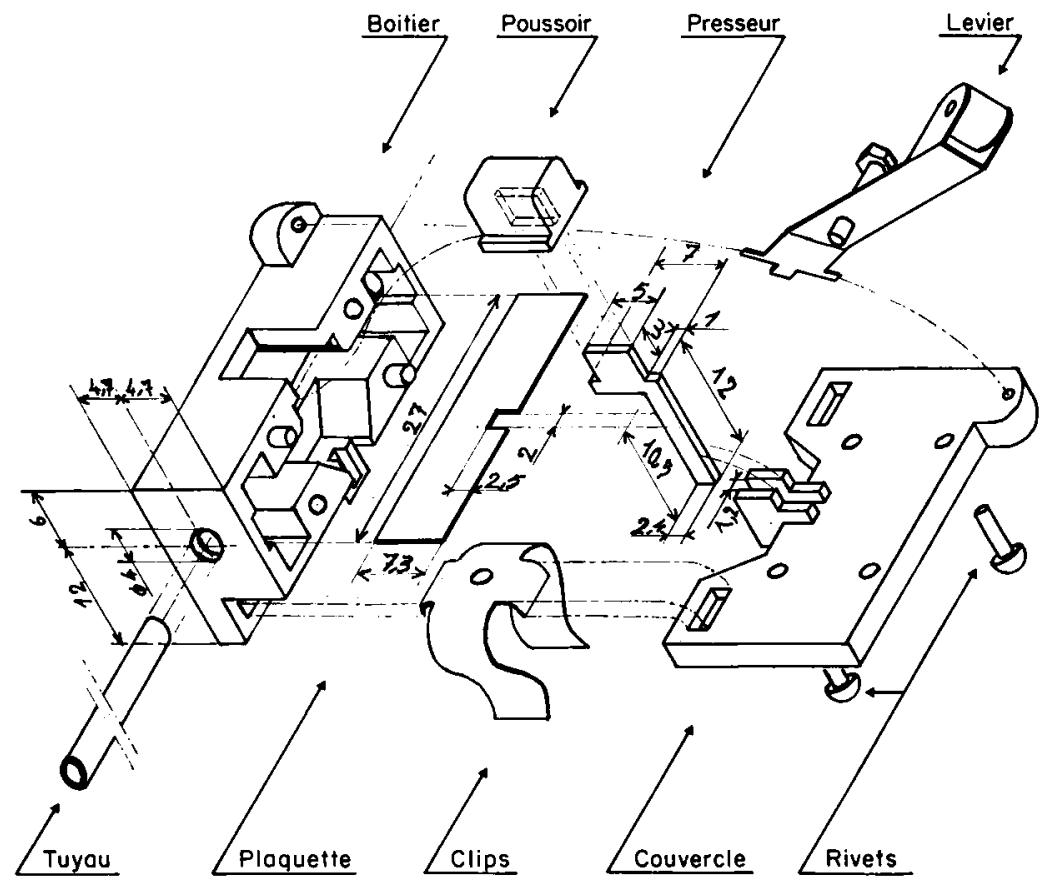

FIG. 2. - Eléments constitutifs de la vanne.

Des vannes de ce type sont utilisées depuis plusieurs années : elles équipent en particulier un analyseur automatique d'acides aminés en service continu depuis 20 mois, sans qu'il ait été nécessaire de changer ou de déplacer les tuyaux en caoutchouc siliconé (fig. 1).

De même, 12 vannes identiques, montées sur un préleveur d'échantillons d'air, ont subi depuis 3 mois de fonctionnement plus de 500000 manœuvres et n'ont nécessité comme entretien que le déplacement du tuyau toutes les 50 à 100000 manœurres (une traction modérée sur le tuyau suffit à réaliser cette opération)*.

Reçu en mars 1977.

Accepté en juillet 1977.

Remerciements. - Nous remercions vivement Monsieur Barbrel (1. N. R. A., Jouy-enJosas) ef ses collaborateurs pour leur participation à la construction de ces vannes.

* Ces réalisations ainsi que celle d'une installation automatique de chromatographie préparative sur résine échangeuse d'ions feront prochainement l'objet de publications séparées. 